\title{
Sobre a necessidade do conceito de fóton
}

On the necessity of photon concept

\author{
Francisco Caruso ${ }^{* 1,2}$, Vitor Oguri ${ }^{2}$ \\ ${ }^{1}$ Centro Brasileiro de Pesquisas Físicas, Coordenação de Física de Altas Energias, 22290-180, Rio de Janeiro, RJ, Brasil. \\ ${ }^{2}$ Universidade do Estado do Rio de Janeiro, Instituto de Física Armando Dias Tavares, 20550-900, Rio de Janeiro, RJ, \\ Brasil.
}

\begin{abstract}
Recebido em 07 de janeiro de 2021. Revisado em 15 de janeiro de 2021. Aceito em 18 de janeiro de 2021.
\end{abstract}
\begin{abstract}
Mostra-se que, apesar de os argumentos de Einstein e Compton terem sido fundamentais para a aceitação do caráter corpuscular da radiação eletromagnética, existem outras abordagens para explicações dos efeitos fotoelétrico e Compton, sem utilizar o conceito de fóton. Nesse contexto, a radiação é tratada como uma onda eletromagnética clássica que interage com o elétron.

Palavras-chave: Fóton; quantização da luz; física moderna; equação de Schrödinger.
\end{abstract}

It is shown that, although Einstein and Compton's arguments were fundamental for the acceptance of the corpuscular character of electromagnetic radiation, there are other approaches able to explain the photoelectric and Compton effects, without using the photon concept. In this context, radiation is treated as a classic electromagnetic wave that interacts with the electron.

Keywords: Photon; light quantization; modern physics; Schrödinger equation.

\section{Introdução}

Os experimentos de difração e interferência da luz realizados por Young, no período de 1800 a 1803, em analogia com os processos de interferência das ondas acústicas, foram interpretados como evidências do caráter ondulatório dos fenômenos luminosos, muito antes da síntese dos fenômenos ópticos e eletromagnéticos feita por Maxwell, em 1865.

A hipótese de que os padrões de interferência observados em um experimento de dupla fenda seriam a corroboração da natureza ondulatória e, portanto, do caráter contínuo dos fenômenos luminosos, se baseia na analogia de Young. Esse argumento é amplamente utilizado, principalmente pelos livros textos, mesmo sabendo-se que os fenômenos acústicos resultam das vibrações coletivas de partículas individuais (átomos ou moléculas) constituintes dos meios materiais.

O conceito de fóton surge também por analogia, em 1905, quando Einstein [1] compara a entropia da radiação de corpo negro com a entropia de um gás ideal em um processo isotérmico. Assim como o gás ideal é um sistema de partículas idênticas e massivas, cada componente monocromática de frequência $\nu$ da radiação seria equivalente a um sistema de partículas idênticas não massivas, cada qual com energia $h \nu$, sendo $h \simeq 6,626 \times 10^{-34}$ J.s a constante de Planck.

A chamada dualidade do comportamento da luz foi estendida às partículas massivas por Louis de Broglie,

*Endereço de correspondência: francisco.caruso@gmail.com em 1924, ao associar, reciprocamente, ao elétron características de um fenômeno ondulatório, como frequência e comprimento de onda. Nesse sentido, experimentos realizados com feixes de elétrons, em 1927, por Davison et al. e Thomson et al., foram interpretados como difração de elétrons pela rede cristalina de um sólido.

Mesmo considerando o elétron como uma partícula, Schrödinger, em 1926, a partir de certos aspectos da analogia de Louis de Broglie, associa ao elétron uma função de onda, e estabelece a equação que rege o comportamento de partículas massivas ao longo do tempo, em uma região do espaço, sob a ação de um campo, no domínio não relativístico 1

Apesar de a hipótese de um quantum de energia ter sido um dos principais fatores da gênese da formulação não relativística da Mecânica Quântica, o conceito de fóton não é necessário para a explicação de fenômenos como os efeitos fotoelétrico e Compton [3], quando a energia $(\epsilon)$ dos elétrons que interagem com a radiação eletromagnética de frequência $\nu$ for bem menor que a energia de repouso do elétron $\left(m c^{2}\right)$. Tal condição pode ser expressa como

$$
\epsilon<h \nu \ll m c^{2}
$$

Interpretações de experimentos baseadas em analogias têm validade heurística, não comprobatória. Mas, apesar de seu caráter não comprobatório, as analogias são

\footnotetext{
${ }^{1}$ Descrições mais detalhadas e referências dos trabalhos originais citados nessa introdução são encontradas nos capítulos 5, 10 e 14 da ref. 2].
} 
importantes no processo de elaboração de modelos, experimentos e teorias científicas.

Na Seção 2 apresenta-se um exemplo de analogia frutífera que resultou em um experimento proposto por Rabi 4, em 1937, o qual originou a técnica de Ressonância Magnética Nuclear.

Deduzida a partir da equação de Schrödinger para a interpretação dos experimentos de ressonância nuclear, a fórmula obtida por Rabi pode ser utilizada na abordagem de qualquer processo físico que envolva a perturbação de um sistema por um campo externo. À luz desse resultado, o caso do efeito fotoelétrico é abordado na Seção 3 e o do efeito Compton, na Seção 4 . Algumas considerações finais são feitas na Seção 5

\section{A fórmula de Rabi}

A ideia de que a absorção da luz incidente em um sistema atômico só ocorre quando a frequência $(\nu)$ for igual a $|\Delta \epsilon| / h$, em que $\Delta \epsilon$ é a diferença entre dois níveis de energia associados aos estados estacionários do sistema, remonta ao modelo de Bohr. Segundo a Mecânica Quântica, diz-se que, devido a uma perturbação externa, o sistema pode realizar transições entre seus autoestados de energia.

Argumento similar foi utilizado por Rabi, ao propor um novo método para a determinação experimental do momento magnético $(\mu)$ associado a um sistema nuclear, a partir de transições ressonantes entre os estados estacionários dos spins de núcleos de um feixe molecular sujeito à ação de uma campo magnético estacionário $\left(\vec{B}_{\circ}\right)$. As transições seriam induzidas por um outro campo magnético dependente do tempo $\left(\vec{B}_{e}\right)$, perpendicular ao campo estacionário, quando a frequência fosse igual à razão entre a diferença entre os níveis de energia dos núcleos, estabelecidos pelo campo estacionário, e a constante de Planck.

A fórmula de Rabi é deduzida a partir do modelo de um sistema regido por uma hamiltoniana $\left(H_{\circ}\right)$ que não depende do tempo, e possui apenas dois estados estacionários $\psi_{1}$ e $\psi_{2}$, correspondentes às energias $\epsilon_{1}$ e $\epsilon_{2}$.

$$
\left\{\begin{array}{l}
H_{\circ} \psi_{1}=\epsilon_{1} \psi_{1} \\
H_{\circ} \psi_{2}=\epsilon_{2} \psi_{2}
\end{array}\right.
$$

Se, em um dado instante $(t=0)$, o sistema sofre a ação de um campo não estacionário, $V^{\prime}(t)$, que acople esses dois estados, tal que

$$
V_{12}^{\prime}=\left(\psi_{1}, V^{\prime} \psi_{2}\right) \neq 0 \quad \text { e } \quad V_{11}^{\prime}=V_{22}^{\prime}=0
$$

em qualquer instante posterior, o estado do sistema, $\Psi(t)$, obedece à equação de Schrödinger,

$$
i \hbar \frac{\partial \Psi}{\partial t}=\left(H_{\circ}+V^{\prime}\right) \Psi
$$

Considerando que a perturbação do campo não estacionário pode induzir transições do sistema entre os dois autoestados de $H_{\circ}$, o estado do sistema perturbado pode ser expresso pela combinação linear de $\psi_{1}$ e $\psi_{2}$,

$$
\Psi(t)=c_{1}(t) \psi_{1} e^{-i \epsilon_{1} t / \hbar}+c_{2}(t) \psi_{2} e^{-i \epsilon_{2} t / \hbar}
$$

sendo que os coeficientes $c_{1}$ e $c_{2}$ dependem do tempo ${ }^{2} \mathrm{e}$ $\left|c_{1}(t)\right|^{2}$ e $\left|c_{2}(t)\right|^{2}=1-\left|c_{1}(t)\right|^{2}$ representam, respectivamente, as probabilidades de ocorrência de cada um dos autoestados $\psi_{1}$ e $\psi_{2}$.

Se $\Psi(0)=\psi_{1}$ é o estado inicial do sistema, $c_{1}(0)=1$ e $c_{2}(0)=0$, e $\left|c_{2}(t)\right|^{2}=P_{1 \rightarrow 2}$ representa também a probabilidade de transição do estado $\psi_{1}$ ao estado $\psi_{2}$, induzida pelo campo perturbativo, após um instante $t$.

Substituindo a expressão (3) na equação de Schrödinger (2), e tendo em conta as eqs. (1), resulta que

$$
\begin{gathered}
i \hbar \dot{c}_{1}(t) \psi_{1} e^{-i \epsilon_{1} t / \hbar}+i \hbar \dot{c}_{2}(t) \psi_{2} e^{-i \epsilon_{2} t / \hbar} \\
=c_{1}(t)\left(H_{\circ}+V^{\prime}\right) \psi_{1} e^{-i \epsilon_{1} t / \hbar} \\
+c_{2}(t)\left(H_{\circ}+V^{\prime}\right) \psi_{2} e^{-i \epsilon_{2} t / \hbar}
\end{gathered}
$$

Levando-se em conta a normalização e a ortogonalidade dos autoestados de $H_{\circ}$, o problema pode ser expresso como um sistema de duas equações diferenciais de 1 a ordem acopladas:

$$
\left\{\begin{aligned}
i \hbar \dot{c}_{1}(t) & =\left(\psi_{1}, V^{\prime} \psi_{2}\right) c_{2}(t) e^{-i\left(\epsilon_{2}-\epsilon_{1}\right) t / \hbar} \\
& =V_{12}^{\prime} e^{-i \omega_{\circ} t / \hbar} c_{2}(t) \\
i \hbar \dot{c}_{2}(t) & =\left(\psi_{2}, V^{\prime} \psi_{1}\right) c_{1}(t) e^{i\left(\epsilon_{2}-\epsilon_{1}\right) t / \hbar} \\
& =V_{21}^{\prime} e^{i \omega_{\circ} t / \hbar} c_{1}(t)
\end{aligned}\right.
$$

em que $\omega_{\circ}=\frac{\left(\epsilon_{2}-\epsilon_{1}\right)}{\hbar}$.

$\mathrm{Na}$ ausência de perturbação $\left(V^{\prime}=0\right)$, de acordo com o estado inicial $\Psi(0)=\psi_{1}, c_{1}(t)=c_{1}(0)=1$ e $c_{2}(t)=$ $c_{2}(0)=0$ são constantes, ou seja, o sistema permanece indefinidamente no autoestado $\psi_{1}$.

Se o termo que acopla os autoestados $\psi_{1}$ e $\psi_{2}$ resulta de um campo que varia harmonicamente com o tempo, com frequência $\omega$, tal que

$$
V^{\prime}(t)=V \cos \omega t \Rightarrow\left\{\begin{array}{l}
V_{12}^{\prime}=\underbrace{\left(\psi_{1}, V \psi_{2}\right)}_{V_{12}} \frac{\left(e^{i \omega t}+e^{-i \omega t}\right)}{2} \\
V_{21}^{\prime}=\underbrace{\left(\psi_{2}, V \psi_{1}\right)}_{V_{12}^{*}} \frac{\left(e^{i \omega t}+e^{-i \omega t}\right)}{2}
\end{array}\right.
$$

os coeficientes devem satisfazer as seguintes equações:

$$
\left\{\begin{array}{l}
i \dot{c}_{1}(t)=\frac{V_{12}}{2 \hbar}\left[e^{-i\left(\omega_{\circ}-\omega\right) t}+e^{-i\left(\omega_{\circ}+\omega\right) t}\right] c_{2}(t) \\
i \dot{c}_{2}(t)=\frac{V_{12}^{*}}{2 \hbar}\left[e^{i\left(\omega_{\circ}-\omega\right) t}+e^{i\left(\omega_{\circ}+\omega\right) t}\right] c_{1}(t)
\end{array}\right.
$$

Longe da chamada condição de ressonância (| $\omega-$ $\left.\omega_{\circ} \mid / \omega_{\circ} \gg 1\right)$, quando as frequências $(\omega)$ são muito

\footnotetext{
2 Esse procedimento, denominado variação dos parâmetros, foi usado por Dirac ao abordar o problema de perturbações dependentes do tempo.
} 
maiores ou muito menores que $\omega_{0}$, ambas as funções exponenciais apresentam oscilações tão rápidas que as variações dos coeficientes são bem pequenas. Assim, $c_{1}(t) \simeq c_{1}(0)=1$ e $c_{2}(t) \simeq c_{2}(0)=0$, ou seja, o sistema praticamente não realiza transições entre os estados $\psi_{1}$ e $\psi_{2}$.

Por outro lado, para frequências $(\omega)$ relativamente próximas a $\omega_{\circ}$, na região de ressonância, o termo que envolve a diferença de frequências determina as taxas de variação dos parâmetros, de modo que as equações para os parâmetros são dadas por:

$$
\left\{\begin{array}{l}
i \dot{c}_{1}(t)=\frac{V_{12}}{2 \hbar} e^{-i \Delta \omega t} c_{2}(t) \\
i \dot{c}_{2}(t)=\frac{V_{12}^{*}}{2 \hbar} e^{i \Delta \omega t} c_{1}(t)
\end{array}\left(\Delta \omega=\left(\omega_{\circ}-\omega\right)\right)\right.
$$

Derivando as equações (5) e eliminando os termos em $c_{1}$, resulta na equação diferencial com coeficientes constantes

$$
\ddot{c}_{2}-i \Delta \omega \dot{c}_{2}+\left(\frac{\omega_{\mathrm{e}}}{2}\right)^{2} c_{2}=0 \quad\left(\omega_{\mathrm{e}}=\left|V_{12}\right| / \hbar\right)
$$

cuja solução geral é dada por

$$
c_{2}(t)=e^{i \Delta \omega t / 2}\left(A_{1} e^{i \Omega t / 2}+A_{2} e^{-i \Omega t / 2}\right)
$$

na qual $\Omega=\sqrt{\omega_{\mathrm{e}}^{2}+\left(\omega_{\circ}-\omega\right)^{2}}$.

De acordo com as condições iniciais,

$$
\left\{\begin{array}{l}
c_{2}(0)=0 \Rightarrow A_{2}=-A_{1} \Rightarrow c_{2}(t)=2 i A_{1} e^{i \Delta \omega t / 2} \operatorname{sen} \frac{\Omega t}{2} \\
c_{1}(0)=1 \Rightarrow\left|\dot{c}_{2}(0)\right|^{2}=\left|\frac{V_{12}}{2 \hbar}\right|^{2}=\left(\frac{\omega_{\mathrm{e}}}{2}\right)^{2}=\left(\Omega A_{1}\right)^{2}
\end{array}\right.
$$

obtém-se a fórmula de Rabi [4, 5] para a probabilidade de ocorrência do estado $\psi_{2}$, quando o estado inicial é $\psi_{1}$,

$$
\left|c_{2}(t)\right|^{2}=P_{1 \rightarrow 2}(t)=\left(\frac{\omega_{\mathrm{e}}}{\Omega}\right)^{2} \operatorname{sen}^{2} \frac{\Omega t}{2}=A(\omega) \operatorname{sen}^{2} \frac{\Omega t}{2}
$$

sendo

$$
\begin{cases}A(\omega)=\frac{\omega_{\mathrm{e}}^{2}}{\omega_{\mathrm{e}}^{2}+\left(\omega_{\circ}-\omega\right)^{2}} & \left(\omega \simeq \omega_{\circ}\right) \\ \Omega=\sqrt{\omega_{\mathrm{e}}^{2}+\left(\omega_{\circ}-\omega\right)^{2}}, & \omega_{\circ}=\frac{E_{2}-E_{1}}{\hbar}, \quad \omega_{\mathrm{e}}=\frac{\left|V_{12}\right|}{\hbar}\end{cases}
$$

Assim, a probabilidade de ocorrência do estado $\psi_{1}$ é dada por

$$
\left|c_{1}(t)\right|^{2}=1-\left|c_{2}(t)\right|^{2}
$$

A probabilidade de ocorrência de cada estado exibe um comportamento oscilatório ao longo do tempo, com frequência $\Omega$ e período $2 \pi / \Omega$. A Fig. 1 mostra que, fora da ressonância, as transições entre os estados ocorrem com baixíssima probabilidade.

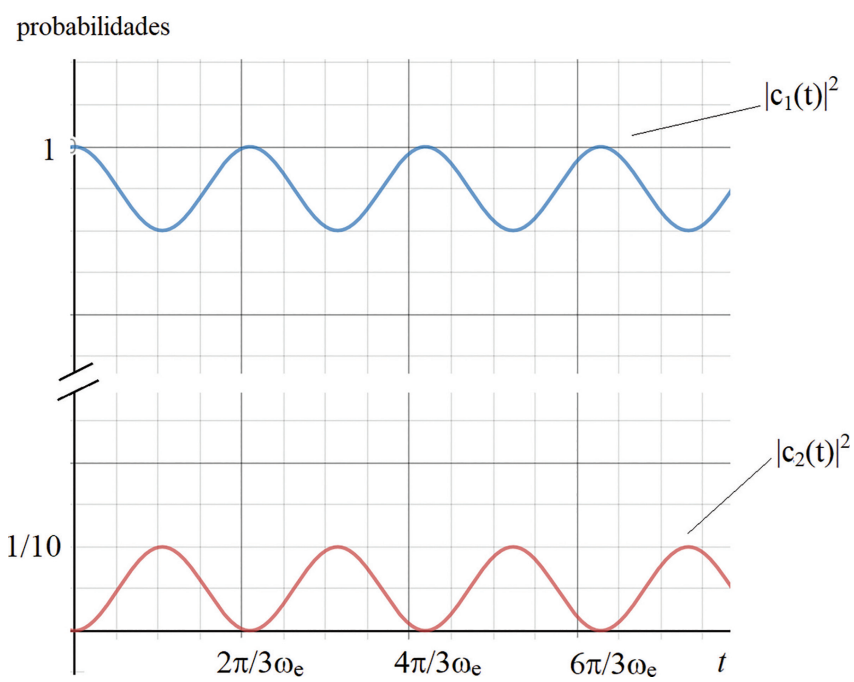

Figura 1: Probabilidades de ocorrência dos estados $\psi_{1}$ e $\psi_{2}$, quando o estado inicial é igual a $\psi_{1}$, para $\left|\omega_{\circ}-\omega\right|=3 \omega_{\mathrm{e}}$, ou seja, $\Omega \gtrsim 3 \omega_{\text {e }}$ e $A(\omega)=1 / 10$.

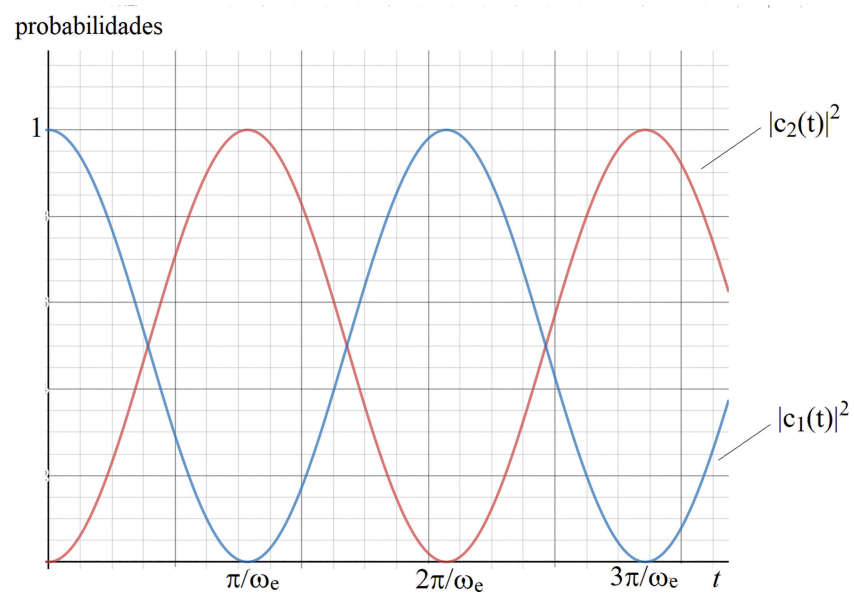

Figura 2: Probabilidades de ocorrência dos estados $\psi_{1}$ e $\psi_{2}$, quando o estado inicial é igual a $\psi_{1}$, para $\omega=\omega_{\circ}$ (ressonância), ou seja, $\Omega=\omega_{\mathrm{e}}=V_{12} / \hbar$ e $A\left(\omega_{\circ}\right)=1$.

Na ressonância $\left(\omega=\omega_{\circ}\right)$, as transições ocorrem em períodos de $\frac{\pi}{\omega_{\mathrm{e}}}=\frac{\pi \hbar}{\left|V_{12}\right|}$, mesmo para um campo externo muito fraco, quando $\omega_{\mathrm{e}} \ll \omega_{\circ} \Rightarrow V_{12} \ll\left(E_{2}-E_{1}\right)$, como mostram as Figs. 2 e 3

Para um sistema de spin $1 / 2$, inicialmente, em um campo magnético constante na direção $\hat{z}\left(\vec{B}_{\circ}=B_{\circ} \hat{z}\right)$,

$$
\begin{gathered}
H_{\circ}=-\vec{\mu} \cdot \vec{B}_{\circ}=-\mu_{z} B_{\circ}=\hbar \underbrace{\gamma B_{\circ}}_{\omega_{\circ}} \frac{\sigma_{z}}{2} \\
\gamma \approx\left\{\begin{array}{l}
10^{8} \mathrm{~T}^{-1} \mathrm{~s}^{-1} \quad \text { (núcleos) } \\
10^{11} \mathrm{~T}^{-1} \mathrm{~s}^{-1} \text { (elétrons) }
\end{array}\right.
\end{gathered}
$$

os dois estados estacionários correspondentes às energias $\epsilon_{2}=\hbar \omega_{\circ} / 2$ e $\epsilon_{1}=-\hbar \omega_{\circ} / 2$ são os autoestados de $\sigma_{z}$, $\psi_{2}=\left(\begin{array}{l}1 \\ 0\end{array}\right)$ e $\psi_{1}=\left(\begin{array}{l}0 \\ 1\end{array}\right)$. 


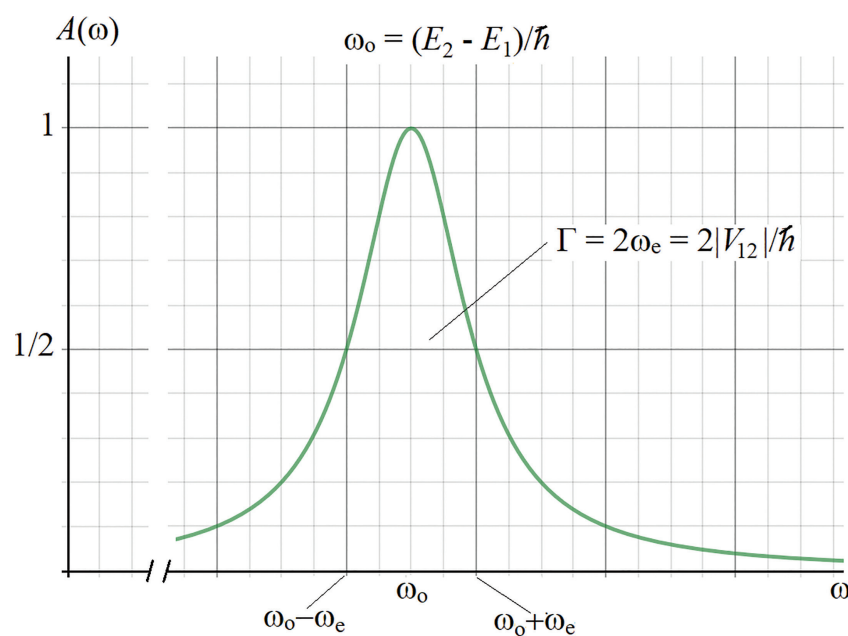

Figura 3: Amplitude da probabilidade de ocorrência do estado $\psi_{2}$, quando o estado inicial é igual a $\psi_{1}$, em função da frequência $\omega$.

Se o sistema é perturbado por um campo magnético harmônico na direção $\hat{x}\left(\vec{B}_{\mathrm{e}}=\hat{x} B_{\mathrm{e}} \cos \omega t\right)$, a energia de interação é dada por

$$
\begin{aligned}
V & =-\vec{\mu} \cdot \vec{B}_{\mathrm{e}}=-\mu_{x} B_{\mathrm{e}} \cos \omega t=\hbar \underbrace{\left(\gamma B_{\mathrm{e}} / 2\right)}_{\omega_{\mathrm{e}}} \sigma_{x} \cos \omega t \\
& =\hbar \omega_{\mathrm{e}}\left(\begin{array}{ll}
0 & 1 \\
1 & 0
\end{array}\right) \cos \omega t
\end{aligned}
$$

e, portanto, o termo de acoplamento ${ }^{3}$ por $V_{12}=V_{21}=$ $\hbar \omega_{\mathrm{e}}=\gamma B_{\mathrm{e}} / 2$.

Para os valores típicos de $B_{\circ}$, deve-se utilizar geradores de $\mathrm{RF}$ e microondas, uma vez que os campos não estacionários devem ter frequências da ordem de $\mathrm{MHz}$ ou GHz.

$$
\begin{aligned}
& B_{\circ} \sim 0,35 \mathrm{~T}-0,7 \mathrm{~T} \\
& \Rightarrow\left\{\begin{array}{l}
\omega_{\circ} \sim 10^{8} B(\mathrm{~T}) \mathrm{s}^{-1} \Rightarrow \nu_{\circ} \sim 10 B(\mathrm{~T}) \mathrm{MHz} \\
\omega_{\circ} \sim 10^{11} B(\mathrm{~T}) \mathrm{s}^{-1} \Rightarrow \nu_{\circ} \sim 10 B(\mathrm{~T}) \mathrm{GHz}
\end{array}\right.
\end{aligned}
$$

Desse modo, ressonâncias magnéticas de spin podem ser induzidas com campos magnéticos não estacionários de intensidades menores do que $10^{-3} \mathrm{~T}$, para os quais

$$
B_{\mathrm{e}} \ll B_{\circ} \sim 1 \mathrm{~T} \Rightarrow \omega_{\mathrm{e}} \ll \omega_{\circ}
$$

O fato de que a ocorrência de transições por ressonância magnética praticamente não depende da intensidade do campo externo e, sim, da frequência, tem certa similaridade com o efeito fotoelétrico. Com efeito, ao incidir radiação eletromagnética sobre a superfície de um

$$
\begin{aligned}
& 3 V_{12}=\hbar \omega_{\mathrm{e}}\left(\begin{array}{ll}
0 & 1
\end{array}\right)\left(\begin{array}{ll}
0 & 1 \\
1 & 0
\end{array}\right)\left(\begin{array}{l}
1 \\
0
\end{array}\right)=\hbar \omega_{\mathrm{e}} \text { e } V_{21}=\hbar \omega_{\mathrm{e}}\left(\begin{array}{ll}
1 & 0
\end{array}\right) \\
& \left(\begin{array}{ll}
0 & 1 \\
1 & 0
\end{array}\right)\left(\begin{array}{l}
0 \\
1
\end{array}\right)=V_{12}=\hbar \omega_{\mathrm{e}}
\end{aligned}
$$

metal, a emissão de elétrons não depende da intensidade da radiação, o fenômeno só ocorre quando a frequência da radiação ultrapassa um certo limite, que depende da natureza do metal.

Uma vez que a fórmula de Rabi expressa a probabilidade de transição entre dois estados estacionários de um sistema ao ser perturbado, ela pode ser utilizada também para a explicação do efeito fotoelétrico sem o conceito de fóton, como foi feito por Lamb e Scully [7, em 1968.

\section{O efeito fotoelétrico}

A fórmula de Rabi, eq. (6), para transições de um estado inicial $\psi_{i}$ com energia $\epsilon_{i}$ para um estado livre final $\psi_{f}$ com energia $\epsilon_{f}$, induzidas por uma pequena perturbação, tal que $\left|V_{i f}\right| \ll\left(\epsilon_{f}-\epsilon_{i}\right)$, pode ser aproximada por [8]

$$
P_{i \rightarrow f}=\left|\frac{V_{f i}}{\hbar}\right|^{2}\left[\frac{\operatorname{sen}\left[\left(\epsilon_{f}-\epsilon_{i}\right) / \hbar-\omega\right] t / 2}{\left(\epsilon_{f}-\epsilon_{i}\right) / \hbar-\omega}\right]^{2}
$$

Esse é o caso da ionização de um átomo quando um elétron absorve energia ao interagir com a luz $(\nu \sim$ $\left.10^{14} \mathrm{~Hz}\right)$.

Esse processo de fotoionização é a origem do efeito fotoelétrico, o qual foi exemplificado por Einstein [1] em 1905, como uma manifestação do caráter corpuscular da luz. No entanto, a partir da eq. (7), o fenômeno pode ser analisado sem evocar o conceito de fóton.

$\mathrm{Na}$ aproximação dipolar, o termo de perturbação do elétron ao ser excitado por um campo eletromagnético que oscila com frequência $\nu$ e amplitude $E_{\circ}$ na direção $\hat{x}$, pode ser expresso com ${ }^{4}$

$$
V^{\prime}(t)=-e \vec{x} \cdot \vec{E}=-e x E_{\circ} \cos 2 \pi \nu t
$$

o que implica $2^{5}$

$$
\left|V_{i f}\right|^{2}=e^{2} E_{\circ}^{2}\left|\left(\psi_{f}, x \psi_{i}\right)\right|^{2}=e^{2} E_{\circ}^{2}\left|x_{f i}\right|^{2}
$$

Assim, segundo a aproximação da fórmula de Rabi, eq. (7), a probabilidade do campo provocar a ionização do átomo é dada pela expressão obtida por Lamb e Scully [7, 9]:

$$
P_{i \rightarrow f}=\left|\frac{e E_{\circ} x_{f i}}{\hbar}\right|^{2}\left[\frac{\operatorname{sen}\left[\left(\epsilon_{f}-\epsilon_{i}\right) / \hbar-\omega\right] t / 2}{\left(\epsilon_{f}-\epsilon_{i}\right) / \hbar-\omega}\right]^{2}
$$

O resultado mostra que a ionização, praticamente, só ocorre quando a condição de ressonância é satisfeita, isto é, a frequência da radiação incidente for igual à diferença

\footnotetext{
4 De acordo com as dimensões atômicas $\left(a \sim 10^{8} \mathrm{~cm}\right)$, para frequências ópticas $\left(\nu \sim 10^{14} \mathrm{~Hz} \rightarrow \lambda \sim 10^{-4} \mathrm{~cm}\right)$, a razão $\frac{a}{\lambda} \approx 10^{-4}$ justifica desprezar as variações espaciais do campo eletromagnético na interação com um átomo.

5 Admitindo-se, para simplificar, que a oscilação do dipolo se efetua na direção do campo. A correção é necessária para o cálculo da distribuição angular do elétron emitido [8].
} 


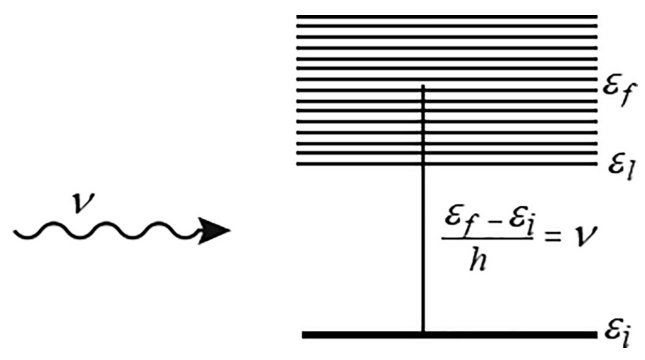

Figura 4: Diagrama da transição de um estado ligado de um elétron com energia $\epsilon_{i}$ para um estado livre com energia $\epsilon_{f}$ ao absorver energia de uma onda luminosa de frequência $\nu . \epsilon_{l}$ é a energia do primeiro estado livre do elétron. A transição só ocorre se $\nu=\left(\epsilon_{f}-\epsilon_{i}\right) / h$.

entre os níveis de energia $\left(\epsilon_{f}-\epsilon_{i}\right)$ dividida pela constante de Planck.

$$
\nu=\frac{\epsilon_{f}-\epsilon_{i}}{h}
$$

A Fig. 4 mostra o processo de transição de um estado ligado de um elétron (em um átomo) com energia $\epsilon_{i}$ para um estado livre com energia $\epsilon_{f}$, devido a ação de um campo eletromagnético de frequência $\nu$.

Tanto na eq. (9) como no diagrama da Fig. 4, a constante de Planck aparece como consequência da equação de Schrödinger, e não associada à energia de um fóton.

Se o átomo pertence a um metal, a diferença entre a energia $\left(\epsilon_{l}\right)$ do primeiro estado livre do elétron e a energia do estado inicial $\left(\epsilon_{i}\right)$, denotada por $\phi=\epsilon_{l}-\epsilon_{i}$, denominada função trabalho, representa a energia mínima necessária para o elétron escapar do metal. A diferença $K=\epsilon_{f}-\epsilon_{l}$ é a máxima energia cinética que o elétron que escapa do metal pode adquirir.

Desse modo, a condição para a ocorrência do fenômeno pode ser expressa também pela equação obtida por Einstein, utilizando o conceito de fóton e a conservação de energia,

$$
\text { (energia do fóton) } h \nu=K+\phi
$$

a qual só é satisfeita se $h \nu \geq \phi$, pois $K>0$.

Uma vez que tanto a intensidade da luz, como a probabilidade de ionização são proporcionais a $\left|E_{\circ}\right|^{2}$, quanto mais intensa for a radiação, maior a quantidade de elétrons emitidos.

Einstein chegou ao conceito de fóton, por argumentos termodinâmicos, ao comparar a entropia da radiação de corpo negro com a entropia de um gás ideal em um processo isotérmico [1, 2]. No entanto, como mostrado por Lamb e Scully [7, 9], o fenômeno pode ser explicado também como consequência da condição de ressonância, sem necessariamente evocar o conceito de fóton, ou seja, não é necessário considerar a quantização da radiação. Nessa abordagem, chamada semiclássica, a radiação é tratada como uma onda eletromagnética clássica, enquanto o espectro de energia do elétron é quantizado.

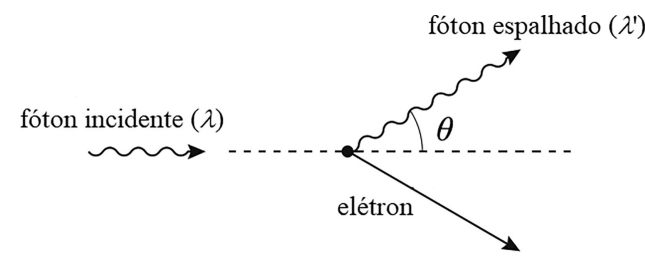

Figura 5: Diagrama de Compton para o espalhamento fótonelétron.

A verificação da fórmula de Einstein, eq. 10, só foi feita por Millikan [10, em 1915, apesar de declaradamente não aceitar o conceito de fóton. Na realidade, por essa época, pouquíssimos pesquisadores aceitavam a hipótese de quantização da radiação.

\section{O efeito Compton}

É bem sabido que, do ponto de vista histórico, a hipótese da existência de fótons só teve ampla aceitação após os experimentos de Compton [3], em 1922, sobre o espalhamento da radiação eletromagnética na faixa dos raios $\mathrm{X}\left(\lambda \sim 10^{-8} \mathrm{~cm}\right)$ por alvos de elementos leves, como o grafite. Nesses experimentos, o comprimento de onda de uma parte dos raios $\mathrm{X}$ espalhados era modificado, contrariando as predições do Eletrodinâmica Clássica.

Aceitando a ideia da existência do fóton, Compton considera que o fenômeno, posteriormente denominado efeito Compton, resulta da colisão de um fóton com um elétron livre 6 Aplicando as leis de conservação de energia e momentum no domínio relativístico, Compton mostra que a variação $(\Delta \lambda)$ do comprimento da radiação é dada por

$$
\Delta \lambda=\left(\frac{2 h}{m c}\right) \operatorname{sen}^{2} \frac{\theta}{2}
$$

em que $m$ é a massa do elétron, $\Delta \lambda=\left(\lambda^{\prime}-\lambda\right)$ é a diferença entre os comprimentos de onda da radiação espalhada $\left(\lambda^{\prime}\right)$ e da incidente $(\lambda)$, e $\theta$ é o ângulo de espalhamento do fóton (Fig. 5).

Assim como o efeito fotoelétrico, as referências [11] e 12 mostram que o efeito Compton pode ser também explicado a partir de uma abordagem semiclássica que não utiliza o conceito de fóton.

\section{Comentários finais}

Procuramos mostrar que, apesar dos argumentos de Einstein e Compton terem sido fundamentais para a aceitação do caráter corpuscular da radiação eletromagnética, existem outras abordagens para explicações

\footnotetext{
${ }^{6}$ Como a energia dos fótons de um feixe de raios $\mathrm{X}$ é muito maior que a energia dos elétrons nos átomos, pode-se considerá-los livres na interação fóton-elétron, mesmo no interior da matéria.
} 

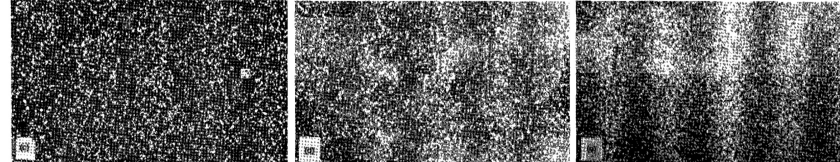

Figura 6: Padrão de interferência, a partir da sucessiva interferência de elétrons individuais, à medida que o número de elétrons vai aumentando.

dos efeitos fotoelétrico e Compton, sem utilizar o conceito de fóton.

Podemos afirmar que a aceitação do conceito de fóton foi consolidada em 1927, a partir do método de quantização proposto por Dirac [13]. No entanto, os experimentos em baixas energias $\sqrt{7}$ que produziram estados da radiação eletromagnética com apenas um fóton só foram realizados em 1986 [14, 15].

Os experimentos de interferência da luz conduzidos por Grangier, Roger e Aspect [15, 16] mostraram padrões de interferência a partir de um único fóton, como afirmado por Dirac [17]:

\section{Each photon then interferes only with itself ${ }^{8}$}

Experimentos de interferência em baixas energias, realizados com elétrons [18] e nêutrons [19] corroboraram também o caráter individual do processo de manifestação de interferência com partículas massivas. A presença de muitas partículas apenas acentua a intensidade do fenômeno, aumentando a estatística do experimento.

A Fig. 6 mostra a evolução do padrão de interferência obtido no experimento de Tonomura et al. [18 com elétrons, em 1989.

Ou seja, contrariando o que é afirmado em muitas abordagens didáticas, a interferência não resulta de interações coletivas das partículas; o padrão de interferência é o resultado do que acontece com partículas isoladas. Tanto as partículas massivas, como os elétrons e os nêutrons, quanto as não massivas, como os fótons, podem interferir com elas próprias.

Assim como o efeito fotoelétrico e o efeito Compton em baixas energias não implicam que a luz seja um sistema de fótons, os fenômenos que apresentam padrões de interferência não devem ser atribuídos à natureza dos sistemas envolvidos. O fato de os experimentos de interferência de feixes de elétrons e nêutrons exibirem padrões similares à interferência da luz proveniente de sua passagem por duas fendas não significa que feixes de elétrons e nêutrons têm o mesmo caráter que a radiação eletromagnética.

A possibilidade de ocorrência do fenômeno de interferência está implícita na linearidade da equação

\footnotetext{
$\overline{7}$ Experimentos nos quais as energias envolvidas são bem menores que as energias de repouso das partículas que participam do processo.

${ }^{8}$ Cada fóton então só interfere com ele próprio.
}

de Schrödinger e das equações de Maxwell em meios lineares. $\mathrm{O}$ fato de o elétron obedecer à equação linear e homogênea de Schrödinger implica propriedades que se manifestam também no comportamento da radiação eletromagnética.

Segundo Dirac 17, essas equações derivam do Princípio da Superposição de Estados, o qual estabelece que quaisquer possíveis estados de um sistema combinados linearmente resultam em um novo possível estado. De modo recíproco, todo estado de um sistema pode ser expresso pela superposição linear de dois ou mais possíveis estados.

Apesar de não serem necessários na análise de alguns processos eletromagnéticos que ocorrem em baixas energias, como o efeito fotoelétrico e o efeito Compton, diagramas como aquele utilizado por Compton são instrumentos mnemônicos que podem facilitar a compreensão de processos que envolvem a interação da radiação eletromagnética com o elétron, ou qualquer sistema de partículas carregadas, como átomos e moléculas.

Além da chamada Óptica Quântica [20, 21, impulsionada a partir da invenção do LASER por trabalhos teóricos 22] e experimentais 23], o conceito de fóton é fundamental em experimentos em Física de Altas Energias para se determinar as distribuições de energia e momentum das partículas emergentes de colisões que envolvem interações eletromagnéticas de partículas eletricamente carregadas 24, 25.

\section{Referências Bibliográficas}

[1] A. Einstein, em: The Collected Papers of Albert Einstein, editado por J. Stachel, D.C. Cassidy, J. Renn e R. Schulmann (Princeton University Press, Oxford, 1989), v. 2.

[2] F. Caruso e V. Oguri, Física Moderna: Origens Clássicas e Fundamentos Quânticos (LTC, Rio de Janeiro, 2016), $2^{\mathrm{a}}$ ed.

[3] A.H. Compton, Phys. Rev. 21, 483 (1923).

[4] I.I. Rabi, J.R. Zacharias, S. Millman e P. Kusch, Phys. Rev. 53, 318 (1938).

[5] J.R.P. Mahon, Mecânica Quântica: desenvolvimento contemporâneo com aplicações (LTC, Rio de Janeiro, 2011).

[6] D.J. Griffiths, Mecânica Quântica (Pearson, São Paulo, 2011).

[7] W.E. Lamb e M.O. Scully, The Photoelectric Effect without Photons, University of Miami, Coral Gables (1968)

[8] J.J. Sakurai e J. Napolitano, Mecânica Quântica Moderna (Bookman, Porto Alegre, 2013), $2^{\mathrm{a}}$ ed.

[9] G. Greenstein e A.G. Zajonc, The Quantum Challenge: Modern Research on the Foundations of Quantum Mechanics (Jones \& Bartllet Pub., Sudbury, 1997).

[10] R.A. Millikan, Phys. Rev. 4, 73 (1914).

[11] J.N. Dodd, Eur. J. Phys. 4, 205 (1983).

[12] J. Strnad, Eur. J. Phys. 7, 217 (1986).

[13] P.A.M. Dirac, Proc. Roy. Soc. A 114, 243 (1927).

[14] C.K. Hong e L. Mandel, Phys. Rev. Lett. 56, 58 (1986). 
[15] P. Grangier e G. Roger, Eur. Phys. Lett. 1, 173 (1986).

[16] C. Ruhla, The Physics of Chance (Oxford University Press, Oxford, 1992).

[17] P.A.M. Dirac, The Principles of Quantum Mechanics (Oxford University Press, Oxford, 1958), $4^{\mathrm{a}}$ ed.

[18] A. Tonomura, J. Endo, T. Matsuda, T. Kawasaki e H. Ezawa, Am. J. Phys. 57, 117 (1989).

[19] A. Zeilinger, R. Gähler, C.G. Shull, W. Treimer e W. Mampe, Rev. Mod. Phys. 60, 1067 (1988).

[20] L. Davidovich, Revista Brasileira de Ensino de Física 37, 4205 (2015).

[21] I. Silva, Revista Brasileira de Ensino de Física 37, 4204 (2015).

[22] R.J. Glauber, Phys. Rev. 130, 2529 (1963).

[23] H.J. Kimble, M. Dagenais e L. Mandel, Phys. Rev. Lett. 39, 691 (1977).

[24] R. Feynman, Quantum Electrodynamics (Perseus Books, New York, 1998).

[25] F. Halzen e A.D. Martin, Quarks \& Leptons: An Introductory Course in Modern Particle Physics (John Wiley \& Sons, Hoboken, 1984). 